\title{
Cross-cultural adaptation and validation of the Romanian International Knee Documentation Committee - subjective knee form
}

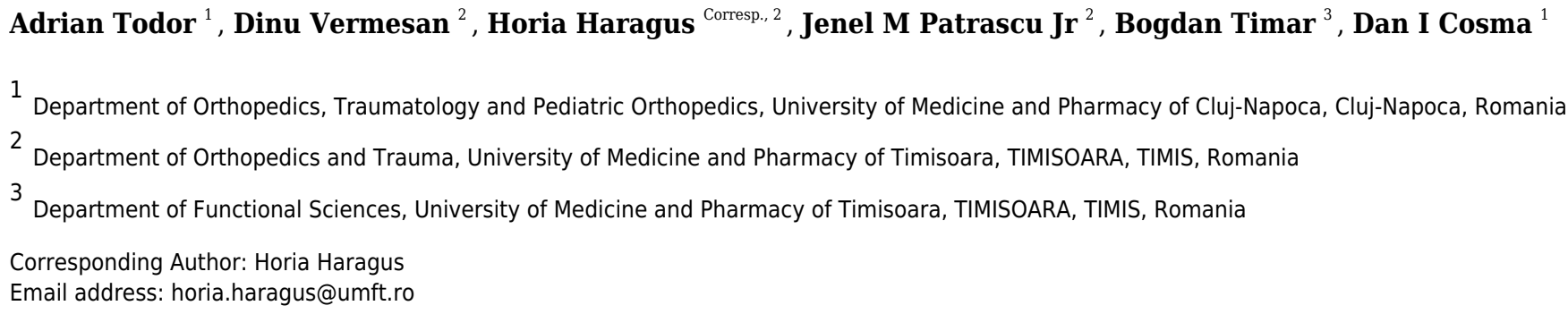

Aim: We aimed to translate and cross-culturally adapt the International Knee Documentation Committee- subjective knee form (IKDC) in Romanian. Method: The original (US) IKDC - subjective knee form was translated according to recommended guidelines. Validity was tested using Spearmans's correlation coefficient between score sand test-retest reproducibility. Reliability and internal consistency were determined using Cronbach's alpha coefficient and intraclass correlation coefficient (ICC). Results: 106 data sets were available for processing. Average age was 52 years and male to female ratio 40:66. 55 subjects repeated the form after an average of 4 days. There were no floor or ceiling effects (range 3.4 to 74.7). There was a strong correlation between the first and repeated administration of the IKDC- subjective knee form $(r=0.816, n=50)$ and moderate compared to Tegner-Lysholm knee rating scale $(r=0.506, n=102)$, KOOSJR (Knee disability and Osteoarthritis Outcome Score for Joint Replacement, $r=-0.622, n=96$ ), EuroqolEQ-5D-5L Index $(r=0.633, n=100)$ and visual analogue scale VAS $(r=0.484, n=99)$. Internal consistency was moderate with Cronbach's alpha $0.611(n=102)$ and ICC 0.611 for average measures (95\% $\mathrm{Cl}$ 0.493-0.713). Conclusion: The Romanian translation of the IKDC - subjective knee form is a valid, consistent and reproducible outcome measure in patients with knee pain and disfunction. 
2 Cross-cultural adaptation and validation of the Romanian International Knee

3 Documentation Committee - subjective knee form

\section{Romanian IKDC}

6

7 Adrian TODOR ${ }^{1}$, Dinu VERMESAN ${ }^{2}$, Horia HARAGUS ${ }^{2 *}$, Jenel M PATRASCUJr ${ }^{2}$, Bogdan

8 TIMAR $^{3}$, Dan I COSMA ${ }^{1}$

10

1. Department of Orthopedics, Traumatology and Pediatric Orthopedics, University of Medicine and Pharmacy of Cluj-Napoca, Cluj-Napoca, Romania

2. Department of Orthopedics and Trauma, University of Medicine and Pharmacy of Timisoara, Timisoara, Timis, Romania

3. Department of Functional Sciences, University of Medicine and Pharmacy of Timisoara, Timisoara, Timis, Romania

17

18

19

20

21

22

23

\section{Abstract}

25

Aim: We aimed to translate and cross-culturally adapt the International Knee Documentation

* Corresponding author: HoriaHaragus

Email: horia.haragus@yahoo.com

ORCID 0000-0001-6801-3471 Committee- subjective knee form (IKDC) in Romanian.

Method: The original (US) IKDC - subjective knee form was translated according to recommended guidelines. Validity was tested using 
32 Results: 106 data sets were available for processing. Average age was 52years and male to 33 female ratio 40:66. 55 subjects repeated the form after an average of 4 days. There were no floor 34 or ceiling effects (range 3.4 to 74.7). There was a strong correlation between the first and

35 36 37 38 39 40

41 repeated administration of the IKDC- subjective knee form $(\mathrm{r}=0.816, \mathrm{n}=50)$ and moderate compared to Tegner-Lysholm knee rating scale $(\mathrm{r}=0.506, \mathrm{n}=102)$, KOOSJR (Knee disability and Osteoarthritis Outcome Score for Joint Replacement, $\mathrm{r}=-0.622$, $\mathrm{n}=96$ ), EuroqolEQ-5D-5L Index $(\mathrm{r}=0.633, \mathrm{n}=100)$ and visual analogue scale VAS $(\mathrm{r}=0.484, \mathrm{n}=99)$. Internal consistency was moderate with Cronbach's alpha $0.611(\mathrm{n}=102)$ and ICC 0.611 for average measures $(95 \% \mathrm{CI}$ 0.493-0.713).

Conclusion: The Romanian translation of the IKDC - subjective knee form is a valid, consistent and reproducible outcome measure in patients with knee pain and disfunction.

Key words: knee joint; osteoarthritis; Meniscectomy;arthroscopy;anterior cruciate ligament; International Knee Documentation Committee;Lysholm Knee Score; Patient Reported Outcome Measures;

\section{Introduction}

Knee pathology is very common. Pain and dysfunction can arise from sports injuries, trauma or degeneration and progress to chronic disability and ultimately osteoarthritis (OA). Quality evaluation of treatment outcomes also takes into account how patients perceive the results. Patient reported outcomes are therefore anintegral part of clinical assessment. They provide insights on the patient's pain relief, performance during activities of daily living, return to sports and level of competitiveness [1-3].

The International Knee Documentation Committee (IKDC) was formed to establish a common ground to evaluate knee function. The IKDCoriginally developed an objective measurement score (clinician completed) to which a subjective knee form(patient completed) was added in 2000. It has since proved to be a commonly used form, withgood psychometric properties. Because it was developed more as a knee specific rather than disease specific outcome scale, the IKDC subjective knee form is versatile, suitable to a wide range of pathologies: sports injuries, anterior cruciate ligament (ACL), meniscus, cartilage and OA [1-3]. 
64 SeveralIKDCsubjective knee form translations are freely available on the American orthopedic

65

66

67

68

69

70

71

72

73

74

75

76

77

78

79

80

81

82

83

84

85

86

87

88

89

90

91

92

93

94

95

96

97

98

society for sports medicine (AOSSM) webpage [4]. It has been translated and culturally adapted in several languages but not Romanian [4-7].

We therefore aimed to perform the translation, cross-cultural adaptation and validation of the International Knee Documentation Committee- subjective knee form (IKDC) in patients with knee pain and disfunction.

11

2

\section{Materials and Methods}

The original 10 questionIKDC-subjective knee formwas retrieved from the developer's website [4]. Response options vary among items: question 6 dichotomizes response into yes/no; questions 1, 4-8, and 9 use 5-point Likert scalesandquestions 2, 3 and 10 use 11-point numerical rating scales. The English (US)IKDCform was translated andculturally validated using the ISPOR (International Society forPharmacoeconomics and Outcomes Research) principles of goodpractice for the translation and cultural adaptation process. The process of translation was straight forward $[\mathbf{8}, \mathbf{9}]$. Two separate translators, proficient in both English and Romanian performed the forward translations with discretionary adnotations. These two forms were reviewed by the authors and we decided on a common form. This later was backward translated from Romanian to English by a native English speaker, proficient in Romanian and the result compared and contrasted to the original English (US) IKDC form. Two authors interviewed 5 subjects for the process of cognitive debriefing. Two issues were raised, one of questionairre construct and one semantic. The IKDC subjective knee form has several items $(1,5,7,8,9$ partial) aimed at diferentiating the ability to perform intense physical activity, such as one is expected to encounter while playing sports. For old and sedentary patients, these items cannot diferentiate well between the normal state of activity and a handicap due to injury or disease. Item 10 was found to be the most complex regarding translation by the authors. It was also found to easily be unclear or confusing to answer by the pretest subjects and possibly requires more attention in order to be accurately answered. Finally the Romanian translated form was proofread by a linguistic expert.

We screenedadultpatients with chronic knee pain and/ or disfunction,evaluated in our University affiliated Emergency clinical county hospital from MarchtoDecember 2018. Diagnosis was supported bypatient history, clinical examination, x-rays, MRI (magnetic resonance imagingwhere available) and arthroscopic exploration (if applicable). Indication for arthroscopy/ meniscectomy was made by the treating physician (orthopedic surgeon) based on current

Peer] reviewing PDF | (2019:10:42043:1:2:NEW 9 Dec 2019) 
99

100

101

102

103

104

105

106

107

108

109

110

111

112

113

114

115

116

117

118

119

120

121

122

123

124

125

126

127

128

129

130

131

guidelines using a standard technique $[\mathbf{1 0 , 1 1}]$. Chronicity was defined as first onset of symptoms at least 4 weeks prior examination in order to maintain homogeneity. Cases with acute trauma, fractures, advanced OA requiring arthroplasty, ACL reconstruction, patellar instability, tumors and septic arthritis were excluded. The study was conducted in accordance with the Declaration of Helsinki and the protocol was approved by the Emergency clinical county hospital 'Pius Brinzeu' Timisoara 'Local ethics committee for scientific research'. All patients gave their informed consent for inclusion before they participated in the study.

The subjects completed Romanian translations of IKDC - subjective knee form and KOOSJR (Knee disability and Osteoarthritis Outcome Score for Joint Replacement) and Euroqol EQ-5D5L Index (converted using the UK tariff) and visual analogue scale (VAS) when seen during clinics $[\mathbf{1 2 , 1 3}$. The examining physician (orthopedic surgeon/ physical therapist) then completed the Tegner-Lysholm knee rating scale [14].

Construct validity was tested using Spearmans'scorrelationcoefficient between the tested scores. Internal consistency was determined using Cronbach's alpha coefficient and test retest reliability using the intraclasscorrelationcoefficient (ICC, two-way mixed effects model) [5-7,9]. For all tests, higher values were associated with better results.Data was analyzed using SPSS v17 statistical software package(SPSS Inc, Chicago, IL, USA).

\section{Results}

One hundred and six (106) data sets were proccesed, out of 110 completed. 19 patients declined participation. 97 underwent knee arthroscopy. Average age was 52 (range 21-83) years and male to female ratio 40:66 (1:1.67). 55 subjects repeated the IKDC - subjective knee form after an average of 4 days (range 1-7). There were no floor or ceiling effects for both IKDC - subjective knee form scores ( $\min 0-\max 100$ ), which ranged from 3.4 to 74.7 for the first and 4.6-74.7 for the second.

Twelve consecutive patients were interviewed and timed at the first completion of the IKDCsubjective knee form. Two required glasses to read the questionnaire. The patients completed the score in an average of 3 minutes and 4 seconds and found it clear and straight forward. Nine estimated that they could complete the questionnaire through mail and phone and even email or tablet with assistance from family members.

There was a strong correlation between the first and repeated administration of the IKDCsubjective knee form $(\mathrm{r}=0.816, \mathrm{n}=50)$ and moderate compared to Tegner-Lysholm knee rating 
$132 \operatorname{scale}(\mathrm{r}=0.506, \mathrm{n}=102)$, KOOSJR $(\mathrm{r}=-0.622, \mathrm{n}=96)$, EQ-5D-5L Index $(\mathrm{r}=0.633, \mathrm{n}=100)$ and VAS $133(\mathrm{r}=0.484, \mathrm{n}=99)$ (see Table. 1, Figures 1 and 2).

134

135 Table. 1

136 Figure. 1

137 Figure.2

138

139 Internal consistency and test-retest reliability were moderate. For the first IKDC-subjective

140 knee form, Cronbach's alpha was $0.611(\mathrm{n}=102)$ and ICC 0.611 for average measures $(95 \% \mathrm{CI}$

141 0.493-0.713). For the retest, Cronbach's alpha was $0.593(\mathrm{n}=55)$ and ICC 0.593 for average

142 measures (95\% CI 0.418-0.734).

143

144

145 Discussion

146 The Romanian translatedandculturallyadaptatedIKDC - subjective knee formproved valid, 147 reliable, consistent and reproducible in patients with non-acute knee pain and disfunction.

148 However, internal consistency and test-retest reliability were moderate, compared to recently 149 published literature regarding IKDC - subjective knee form translations: TurkishCronbach's 0.89 150 and ICC 0.91; Greek Cronbach's 0.87 and ICC 0.95; Chinese Cronbach's 0.87 and ICC 0.97 [5151 7].

152 Most of our valid entries (91.5\%) were patients who underwent knee arthroscopy. Out of them, 153 by far the main indication was meniscectomy. Treatment of symptomatic knees with meniscus 154 tears can be controversial, however it is still one of the most routinely performed orthopedic 155 surgical procedure $[\mathbf{3 , 1 0 , 1 1}]$. Cartilage surgery is still under development, with cautiously 156 optimistic predictions for the future [15]. The transition from cartilage defects and meniscus tears 157 to early OA is many times difficult. For incipient knee degeneration, definition, symptoms, 158 magnetic resonance imaging and outcome measures are not yet standardized. Nevertheless, for 159 this subpopulation the IKDC - subjective knee form, the KOOSJR, Tegner-Lysholm knee rating 160 scale and Euroqol EQ-5D-5L are among the most commonly used and supported patient reported 161 outcomes $[\mathbf{1 , 3 , 1 6}]$. 
162 The ACL is arguably the most commonly reconstructed ligament in the human body. It is the

163

164

165

166

167

168

169

170

171

172

173

174

175

176

177

178

179

180

181

182

183

184

185

186

187

188

189

190

191

192

193

194

195

main stabilizer against anterior tibial translation, with functional importance in sports. The aforementioned outcome measures apply also in cruciate ligament reconstruction $[\mathbf{2 , 3 , 1 7}]$. We deliberately excluded ACL surgeries to maintain group homogeneity and target the non-acute pain, meniscus and early degeneration subpopulation.

Our study has several limitations. We did not use the entireIKDCquestionnaire, nor the complete KOOS (Knee disability and Osteoarthritis Outcome Score). Instead, we opted for the TegnerLysholm knee rating scale, a versatile and simple outcome measure, widely used in sports and arthroscopy, that can be completed both by the clinician as well as the patient $[2,3]$. The shorter version of the KOOS developed for joint replacement was also favored as a shorter, simpler form for early knee OA. We based our decision on the considerable overlap of patients with early radiographic OA with those with advanced degeneration $[1,12,16]$. For the general wellbeing, the Euroqol EQ-5D-5L is a free, widely used patient questionnaire $[\mathbf{1 , 1 8}]$. However, to quantify impairment we had too use the UK tariff as the best approximation since there is no conversion available for Romania. In the original development study of the IKDC, the authors used the Short Form 36 (SF-36) subscales as comparators, which became the standard for subsequent validations [3-7]. Compared to the 5 item EQ-5D-5L, the SF-36, commercially administered by the RAND Corporation (Santa Monica, CA, USA) has 36, divided into 8 sections. The issues listed above probably had 2 effects: they significantly reduced the effort required to fill out the forms at the cost of decreasing the validity correlation strength. We felt this to be a fair trade as well as maintain a current trend towards the reduction of item number deemed relevant in regularly used patient reported outcomes. It is likely that simplified versions of highly referenced scores and computer adapted technology will usher in the implementation of patient reported outcomes from the research field to routine clinical practice $[\mathbf{1 2 , 1 8 , 1 9 ]}$.

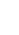

7

\section{Conclusions}

The Romanian translation of the IKDC - subjective knee form is a valid, consistent and reliable outcome measure in patients with knee pain and disfunction. However, internal consistency and test-retest reliability were moderate compared to published literature.

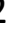

Acknowledgements: Bogdan Deleanu, MD, PhD; IoanaGeorgescu, MD; Andrei Ghiorghitoiu, MD; Musat Roxana, Med Stud. 
198 References

199 1. Emery CA, Whittaker JL, Mahmoudian A, et al 2019. Establishing outcome measures in early knee

200

201

202

203

204

205

206

207

208

209

210

211

212

213

214

215

216

217

218

219

220

221

222

223

224

225

226

227

228

229

230

231

232

233

234

235

236

2. Ahmad SS, Meyer JC, Krismer AM, et al 2017. Outcome measures in clinical ACL studies: an analysis of highly cited level I trials. Knee Surg Sports TraumatolArthrosc. 25:1517-27. doi: 10.1007/s00167-016-4334-4.

3. Grevnerts HT, Terwee CB, Kvist J 2015. The measurement properties of the IKDC-subjective knee form. Knee Surg Sports TraumatolArthrosc. 23:3698-706. doi: 10.1007/s00167-014-3283-z.

4. AOSSM website 2019. Available at https://www.sportsmed.org/aossmimis/Staging/Research/IKDC_Forms.aspx(accessed 4 Sept 2019).

5. Çelik D, Coşkunsu D, KiliÇoğlu Ö, Ergönül Ö, Irrgang JJ 2014. Translation and cross-cultural adaptation of the international knee documentation committee subjective knee form into Turkish. $J$ Orthop Sports Phys Ther. 44:899-909. doi: 10.2519/jospt.2014.4865.

6. Koumantakis GA, Tsoligkas K, Papoutsidakis A, Ververidis A, Drosos GI 2016. Cross-cultural adaptation and validation of the International Knee Documentation Committee Subjective Knee Form in Greek. J OrthopTraumatol. 17:123-9. doi: 10.1007/s10195-015-0362-y.

7. Huang CC, Chen WS, Tsai MW, Wang WT 2017. Comparing the Chinese versions of two kneespecific questionnaires (IKDC and KOOS): reliability, validity, and responsiveness. Health Qual Life Outcomes. 15:238. doi: 10.1186/s12955-017-0814-6.

8. Wild D, Grove A, Martin M, Eremenco S, McElroy S, Verjee-Lorenz A, Erikson P 2005 ISPOR Task Force for Translation and Cultural Adaptation. Principles of good practice for the translation and cultural adaptation process for patient-reported outcomes (PRO) measures: report of the ISPOR Task Force for translation and cultural adaptation. Value Health. 8:94-104.

9. Haragus H, Prejbeanu R, Poenaru DV, Deleanu B, Timar B, Vermesan D 2018. Cross-cultural adaptation and validation of a patient-reported hip outcome score. IntOrthop. 42:1001-6. doi: 10.1007/s00264-017-3742-5.

10. Beaufils P, Becker R, Kopf S, Matthieu O, Pujol N 2017. The knee meniscus: management of traumatic tears and degenerative lesions. EFORT Open Rev. 2:195-203. doi: 10.1302/20585241.2.160056.

11. Todor A, Caterev S, Nistor DV 2016. Outside-In Deep Medial Collateral Ligament Release During Arthroscopic Medial Meniscus Surgery. Arthrosc Tech. 5:e781-85.

12. Lyman S, Lee YY, Franklin PD et al 2016. Validation of the KOOS, JR: a short-form knee arthroplasty outcomes survey. ClinOrthopRelat Res; 474:1461-71.

13. EuroQol website 2019. Available at http://www.euroqol.org/about-eq-5d/how-to-use-eq-5d.html (accessed 4 Sept 2019).

14. Orthopaedicscores website 2019. Available at https://www.orthopaedicscore.com/scorepages/tegner_lysholm_knee.html (accessed 4 Sept 2019).

15. Fodor P, Solyon A, Fodor R, et al 2018. Role of the Biomimetic Scaffolds in the Regeneration of Articular Tissue in Deep Osteochondral Defects in a Rabbit Model. Rev. Chim. (Bucharest). 1:201-7. 
237 238 239

240

241

242

243

244

245

246

247

248

249

250

\section{Figure legends}

252 Fig. 1 Moderate correlation between IKDC (International Knee Documentation Committee 253 subjective knee form) andTegner-Lysholmknee rating scale.

254 Fig. 2 Moderate correlation between IKDC (International Knee Documentation Committee 255 subjective knee form) and EQ-5D-5L Index. 


\section{Table $\mathbf{1}$ (on next page)}

Spearman's rho correlation coefficients between the tested scores

Correlations between the two IKDC (International Knee Documentation Committee subjective knee form) scores, Tegner-Lysholm knee rating scale, KOOSJR (Knee disability and Osteoarthritis Outcome Score for Joint Replacement) and EQ-5D-5L Index and VAS (Visual analog scale). Presented as coefficient/ $p$ value and number of subjects. ${ }^{* *}$ Correlation is significant at the 0.01 level (2-tailed). 
1 Table.1 Spearman's rho correlation coefficients between the tested scores

\begin{tabular}{|c|c|c|c|c|c|c|}
\hline & IKDC & ikdc2 & Lysholm & KOOSJR & EQ-5D-5L & VAS \\
\hline \multirow{3}{*}{ IKDC } & 1.000 & $.816^{* *}$ & $.506^{* *}$ & $-.622^{* *}$ & $.633^{* *}$ & $.484^{* *}$ \\
\hline & . & .000 & .000 & .000 & .000 & .000 \\
\hline & 103 & 50 & 102 & 96 & 100 & 99 \\
\hline \multirow{3}{*}{ ikdc2 } & $.816^{* *}$ & 1.000 & $.392^{* *}$ & $-.670^{* *}$ & $.586^{\star *}$ & $.568^{* *}$ \\
\hline & .000 & . & .004 & .000 & .000 & .000 \\
\hline & 50 & 54 & 53 & 48 & 50 & 49 \\
\hline \multirow{3}{*}{ Lysholm } & $.506^{* \star}$ & $.392^{* *}$ & 1.000 & $-.546^{* *}$ & $.513^{* *}$ & $.436^{* *}$ \\
\hline & .000 & .004 & . & .000 & .000 & .000 \\
\hline & 102 & 53 & 105 & 95 & 99 & 98 \\
\hline \multirow{3}{*}{ KOOSJR } & $-.622^{* *}$ & $-.670^{* *}$ & $-.546^{* *}$ & 1.000 & $-.562^{* *}$ & $-.567^{* *}$ \\
\hline & .000 & .000 & .000 & . & .000 & .000 \\
\hline & 96 & 48 & 95 & 96 & 96 & 92 \\
\hline \multirow{3}{*}{ EQ-5D-5L } & $.633^{* *}$ & $.586^{\star *}$ & $.513^{* *}$ & $-.562^{* *}$ & 1.000 & $.591^{\star *}$ \\
\hline & .000 & .000 & .000 & .000 & . & .000 \\
\hline & 100 & 50 & 99 & 96 & 100 & 96 \\
\hline \multirow{3}{*}{ VAS } & $.484^{* *}$ & $.568^{* *}$ & $.436^{* *}$ & $-.567^{* *}$ & $.591^{\star *}$ & 1.000 \\
\hline & .000 & .000 & .000 & .000 & .000 & $\cdot$ \\
\hline & 99 & 49 & 98 & 92 & 96 & 99 \\
\hline
\end{tabular}

2 Table. 1 Correlations between the two IKDC (International Knee Documentation Committee 3 subjective knee form) scores, Tegner-Lysholm knee rating scale, KOOSJR (Knee disability and 4 Osteoarthritis Outcome Score for Joint Replacement) and EQ-5D-5L Index and VAS (Visual 5 analog scale). Presented as coefficient/ $p$ value and number of subjects. ${ }^{* *}$ Correlation is 6 significant at the 0.01 level (2-tailed). 
Figure 1

Correlation between IKDC and Tegner-Lysholm

Moderate correlation between IKDC (International Knee Documentation Committee subjective knee form) and Tegner-Lysholm knee rating scale.

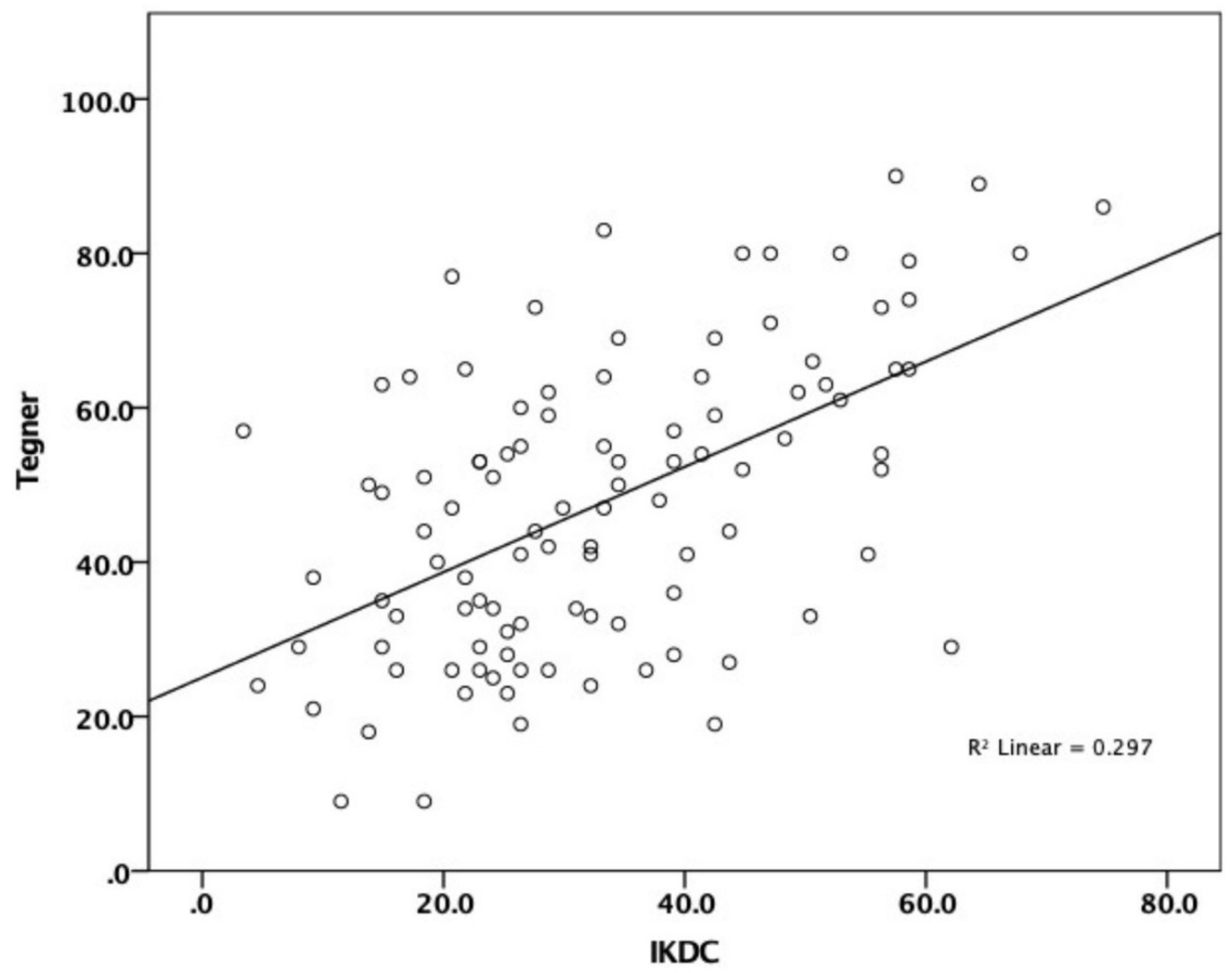


Figure 2

Correlation between IKDC and EQ-5D Index

Moderate correlation between IKDC (International Knee Documentation Committee subjective knee form) and EQ-5D-5L Index

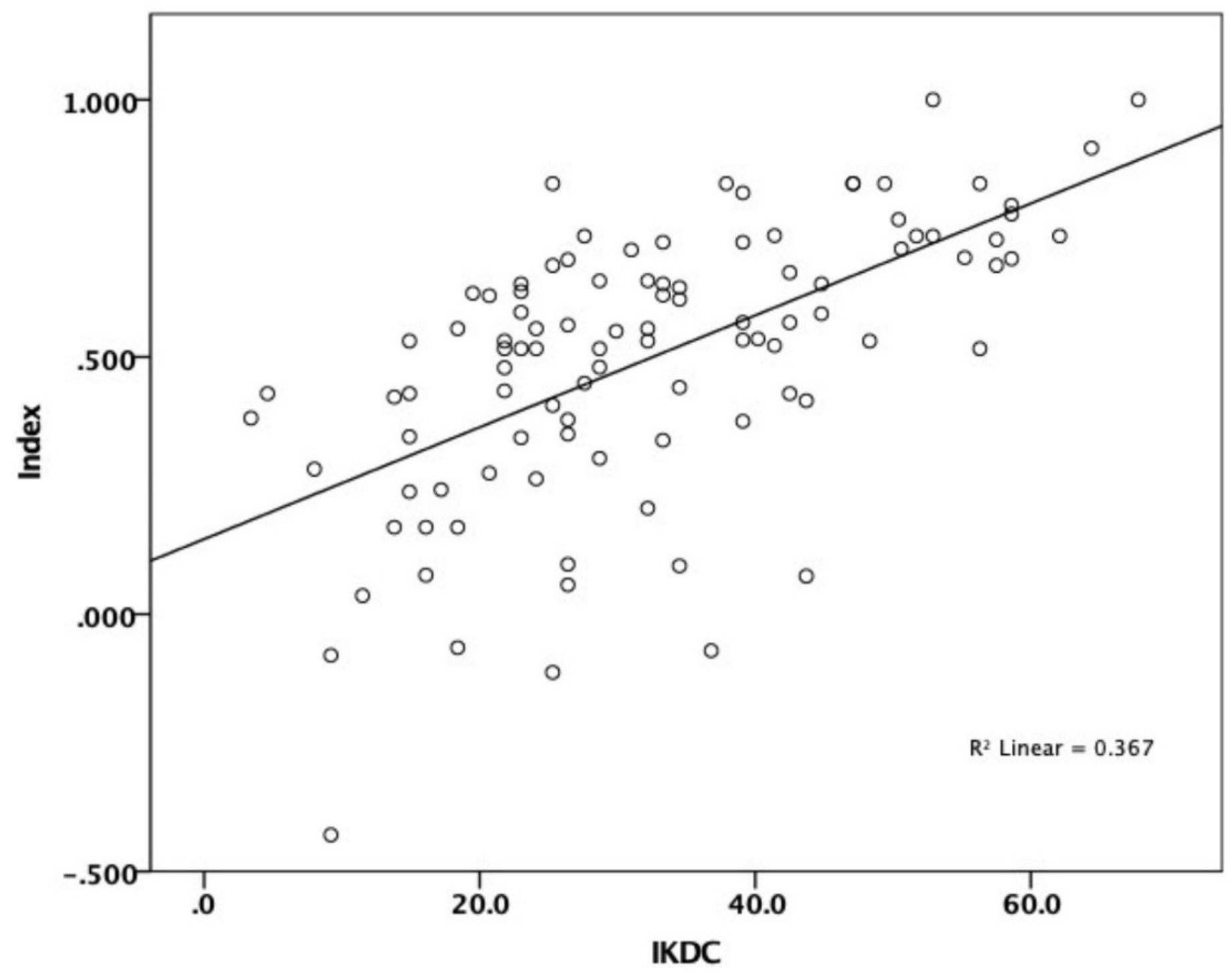

